\title{
The development or worsening of hypertension after transcatheter aortic valve replacement (TAVR) improves short-term and long-term patient outcomes
}

\author{
Brent J Klinkhammer, ${ }^{1}$ Cornelius M Dyke, ${ }^{1,2}$ Thomas A Haldis ${ }^{2}$
}

${ }^{1}$ Internal Medicine, University of North Dakota School of Medicine and Health Sciences, Grand Forks, North Dakota, USA ${ }^{2}$ Cardiology, Sanford Health Heart Hospital, Fargo, North Dakota, USA

Correspondence to Dr Brent J Klinkhammer, University of North Dakota School of Medicine and Health Sciences, Grand Forks, ND 58202, USA; brent. klinkhammer@unmc.edu

Received 26 December 2017 Revised 19 April 2018 Accepted 20 April 2018
Check for updates

To cite: Klinkhammer BJ Dyke CM, Haldis TA. Heart Asia 2018;10:e010994. doi:10.1136/

heartasia-2017-010994

\section{ABSTRACT}

Objectives In patients with symptomatic aortic valve disease who are at intermediate to high risk for open surgical aortic valve replacement, transcatheter aortic valve replacement (TAVR) decreases overall mortality and improves quality of life. Hypertension (HTN) after TAVR has been associated with improved cardiac function and short-term survival but its effect on survival over 1 year is unclear. Our study aims to evaluate the effect of HTN following TAVR on short-term and long-term clinical and echocardiographic outcomes

Methods A retrospective chart review case-control study of 343 consecutive patients who underwent TAVR between August 2012 and November 2016 was performed to elucidate the relationship between HTN and post-TAVR outcomes.

Results 193 patients who underwent TAVR (56.2\%) developed or had a worsening of their HTN after TAVR. The development of post-TAVR HTN was associated with a significantly better overall survival at 1 year ( $89 \%$ vs $67 \%, p<0.001)$ and 2 years ( $72 \%$ vs $46 \%, p=0.002$ ). Patients with increased blood pressure also had a significant lower in hospital cardiovascular mortality ( $1 \%$ vs $12 \%, p<0.001)$. However, the development or worsening of their HTN after TAVR was associated with an increase in heart failure (HF) exacerbations and diuretic use.

Conclusions The development or worsening of HTN after TAVR is associated with improved overall survival despite an increase in postprocedural HF exacerbations and antihypertensive medication utilisation. The outcomes of this study could be important in postoperative management of patients who underwent TAVR.

\section{INTRODUCTION}

Transcatheter aortic valve replacement (TAVR) has been a major advancement in the treatment of symptomatic aortic valve disease in patients at intermediate or high risk for surgical complications. In this population, outcomes after TAVR have consistently shown benefit over non-surgical medical treatment, as well as improvements in hospitalisation rate, patient symptoms and overall quality of life. ${ }^{12}$ Despite the $44 \%$ improvement in overall survival versus medical management, the 1-year and 2-year overall mortality remains elevated at $24.2 \%$ and $43.3 \%$, respectively, in the highest risk patients. ${ }^{13}$

There has been little published data on validated postprocedural prognostic factors that could be easily applied to tailor post-TAVR management based on the patient's clinical and haemodynamic response to TAVR. Postoperative factors associated with an increased risk of death after TAVR include acute kidney injury, major bleeding complications, postprocedural cardiac tamponade, elevated creatine kinase-myocardial band (CK-MB), postTAVR paravalvular leak, left-ventricular stroke volume index at discharge, severe pulmonary HTN, postinterventional myocardial infarction, stroke, pulmonary embolism or systemic inflammatory response syndrome (SIRS). ${ }^{4-14}$ New onset atrial fibrillation following TAVR and post-TAVR permanent pacemaker implantation have been associated with a poor prognosis, but not consistently with overall mortality. ${ }^{15} 16$

In 2013, Perlman et al published a study of 105 patients who underwent TAVR which demonstrated improved postprocedural outcomes, but not mortality in patients who developed new onset HTN or had increased blood pressure (BP) during the first five postprocedural days. ${ }^{17}$ Perlman et al concluded that increased BP after TAVR indicates a relative improvement of cardiac function and suggests a better overall haemodynamic response to TAVR, but without an improvement in survival. The authors also noted the contradictory nature of an association between increased BP and improved outcomes, especially in elderly patients with the most arterial stiffness. In 2017, Lindman et al showed that higher systolic BP is associated with improved overall, but this study was limited to 1 year post-TAVR. ${ }^{18}$ In this study, we extended the period of HTN and outcome monitoring to better associate new onset or worsening HTN with improved outcomes.

\section{METHODS}

A hospital-based, single institution case-control study was conducted using data from one large single-centre, tertiary care referral centre. We performed a retrospective chart review of 343 consecutive patients who underwent TAVR at Sanford Health in Fargo, ND from August 2012 to November 2016. The last date of data acquisition was 1 April 2017. The entire cohort was divided in two groups: the patients who developed new onset HTN or increased systolic or diastolic BP meeting study criteria within a 1 -year postoperative follow-up period or by the last date of data acquisition, and those who did not meet this criteria. Patients with HTN needed to meet at least one of the following three criteria: (1) a sustained (48 hours) systolic pressure $140 \mathrm{~mm} \mathrm{Hg}$ or diastolic pressure $90 \mathrm{~mm} \mathrm{Hg}$ that was not present at baseline, (2) a need to increase the dosage of an antihypertensive 
Table 1 Baseline characteristics

\begin{tabular}{|c|c|c|c|}
\hline & Increased BP (193) & Stable BP (150) & $P$ value \\
\hline Age & $81(74-85)$ & $81(74-86)$ & 0.802 \\
\hline Male sex & $96(50)$ & $96(64)$ & 0.009 \\
\hline $\mathrm{BMI}$ & $29.55(26.43-34.29)$ & $29.66(26.05-32.99)$ & 0.668 \\
\hline Caucasian race & $191(99)$ & $149(99)$ & 1.000 \\
\hline EuroSCORE $(\%)$ & $7.09(4.50-11.28)$ & $6.96(4.77-10.08)$ & 0.523 \\
\hline STS risk score (\%) & $5.4(3.6-8.8)$ & $6.1(4.0-9.1)$ & 0.178 \\
\hline Preprocedural HTN & $172(89)$ & $130(87)$ & 0.506 \\
\hline Preprocedural CAD & $145(75)$ & $107(71)$ & 0.461 \\
\hline Baseline ejection fraction $<40 \%$ & $23(12)$ & $26(17)$ & 0.165 \\
\hline $\begin{array}{l}\text { Preprocedural NYHA } \\
\text { class III or IV symptoms }\end{array}$ & $85(44)$ & $67(45)$ & 0.913 \\
\hline Preprocedural DM & $70(36)$ & $52(35)$ & 0.820 \\
\hline Prior stroke/TIA & $27(14)$ & $12(8)$ & 0.089 \\
\hline Preprocedural atrial fibrillation & $54(28)$ & $50(33)$ & 0.290 \\
\hline Preprocedural eGFR $<60 \mathrm{~mL} / \mathrm{min}$ & $95(49)$ & $69(46)$ & 0.587 \\
\hline Preprocedural AAA & $18(9)$ & $20(13)$ & 0.298 \\
\hline $\begin{array}{l}\text { Preprocedural carotid artery stenosis }>50 \% \text { or prior } \\
\text { CEA }\end{array}$ & $54(28)$ & $39(26)$ & 0.715 \\
\hline Preprocedural dyslipidaemia & $171(89)$ & $134(89)$ & 0.864 \\
\hline Prior $\mathrm{CABG}$ & $57(30)$ & $40(27)$ & 0.629 \\
\hline Prior $\mathrm{PCl}$ & $65(34)$ & $61(41)$ & 0.214 \\
\hline Prior permanent pacemaker & $24(12)$ & $17(11)$ & 0.867 \\
\hline Prior aortic valvuloplasty & $31(16)$ & $28(19)$ & 0.565 \\
\hline
\end{tabular}

Values are median (IQR) or $\mathrm{n}(\%)$.

AAA, abdominal aortic aneurysm; BMI, body mass index; BP, blood pressure; CABG, coronary artery bypass graft; CAD, coronary artery disease; CEA, carotid endarterectomy; DM, diabetes

mellitus; eGFR, estimated glomerular filtration rate HTN, hypertension; NYHA, New York Heart Association; PCI, percutaneous coronary intervention; STS, TIA, transient ischaemic attack.

drug by twofold or more from the preprocedural dose to achieve control of systemic BP or (3) a need to add an additional antihypertensive medicine to the preprocedural regimen in order to achieve control of systemic BP. Primary outcomes were overall survival at 1 month, 6 months, 1 year and 2 years post-TAVR. Secondary outcomes were procedural complications, post-TAVR permanent pacemaker implantation, major adverse cardiovascular and cerebrovascular events (MACCE) defined as death from any cause, myocardial infarction, rehospitalisation for stroke, cardiovascular mortality, myocardial infarction, stroke/ TIA, heart failure (HF) exacerbation or rehospitalisation for any reason in defined time periods. Preprocedural and postprocedural echocardiographic data along with baseline, immediate postprocedural and 1-year HTN data were also compared. The clinical outcomes were assessed in accordance with the standardised endpoint definitions for TAVR of the Valve Academic Research Consortium-2. ${ }^{19}$ HF exacerbation was defined as a gradual or rapid change in HF signs and symptoms resulting in a need for a change in therapy or hospitalisation.

A waiver for informed consent was granted due to the retrospective nature of the study. The Fisher's exact test was performed to determine statistical significance of categorical data and t-test or Wilcoxon two-sample test was used to determine the statistical significance continuous variables. All $p$ values were two-sided, and $\mathrm{p}$ values $<0.05$ were considered significant.

\section{RESULTS}

A total of 193 of the 343 patient met study criteria for postTAVR HTN. Baseline characteristics for both groups are given in table 1 . The two study cohorts were well matched, except for an increased proportion of female subjects in the cohort meeting the post-TAVR HTN criteria. Significant comorbidities existed in both groups. The overall prevalence of preprocedural HTN was $88 \%$ in the entire cohort. The mean age of the entire cohort was 79.2 years. Procedural characteristics for both groups are given in table 2. There were no statistical differences in the approach used for TAVR, however there was small, but statistical significantly increase in the utilisation of the first-generation Edwards Sapien valve in the cohort that met study post-TAVR HTN criteria. Preprocedural and postprocedural echocardiographic data are given in table 3 . No sustained differences in ejection fraction or stroke volume were found. Primary and secondary outcomes are detailed in table 4. Overall survival for the entire study cohort was $79.7 \%$ at 1 year and $60.5 \%$ at 2 years. Patients

\begin{tabular}{|c|c|c|c|}
\hline & Increased BP & Stable BP & $P$ value \\
\hline \multicolumn{4}{|l|}{ Approach } \\
\hline Transfermoral & $154(80)$ & $125(83)$ & 0.485 \\
\hline Transapical & $31(16)$ & $19(13)$ & 0.442 \\
\hline Transaortic & $5(3)$ & $2(1)$ & 0.474 \\
\hline Trans-subclavian & $2(1)$ & $4(3)$ & 0.410 \\
\hline Transcaval & $1(1)$ & $0(0)$ & 1.000 \\
\hline Mean LOS after TAVR (days) & $5.2(8.6)$ & $4.2(4.7)$ & 0.214 \\
\hline \multicolumn{4}{|l|}{ Valve type } \\
\hline First generation Sapien & $60(31)$ & $32(21)$ & 0.049 \\
\hline Sapien XT & $29(15)$ & $23(15)$ & 1.000 \\
\hline Sapien S3 & $62(32)$ & $52(35)$ & 0.645 \\
\hline First-generation CoreValve & $37(19)$ & $37(25)$ & 0.236 \\
\hline CoreValve evolute & $5(3)$ & $6(4)$ & 0.544 \\
\hline Mean valve size (mm) & $25.8(2.7)$ & $26.3(2.7)$ & 0.079 \\
\hline
\end{tabular}


Table 3 Echocardiographic data

\begin{tabular}{|c|c|c|c|}
\hline & Increased BP & Stable BP & $P$ value \\
\hline \multicolumn{4}{|l|}{ Baseline } \\
\hline Aortic valve area $(\mathrm{VTI})\left(\mathrm{cm}^{2}\right)$ & $0.87(0.31)$ & $0.89(0.43)$ & 0.984 \\
\hline Peak aortic velocity $(\mathrm{cm} / \mathrm{s})$ & $417(62.3)$ & $412(70.8)$ & 0.777 \\
\hline Peak aortic gradient $(\mathrm{mm} \mathrm{Hg})$ & $71.3(19.9)$ & $70.4(21.7)$ & 0.684 \\
\hline Mean aortic gradient $(\mathrm{mm} \mathrm{Hg})$ & $45.0(12.7)$ & $44.4(13.3)$ & 0.909 \\
\hline Ejection fraction (\%) & $58.8(11.7)$ & $55.8(13.8)$ & 0.056 \\
\hline Stroke volume $(\mathrm{mL})$ & $86.4(21.8)$ & $85.8(20.1)$ & 0.864 \\
\hline Moderate aortic regurgitation (\%) & $44(23)$ & $22(15)$ & 0.055 \\
\hline Severe aortic regurgitation (\%) & $7(4)$ & $4(3)$ & 0.760 \\
\hline Moderate mitral regurgitation (\%) & $46(24)$ & $31(21)$ & 0.515 \\
\hline Severe mitral regurgitation (\%) & $7(4)$ & $5(3)$ & 1.000 \\
\hline \multicolumn{4}{|l|}{24 hours post-TAVR } \\
\hline Aortic valve area (VTI) $\left(\mathrm{cm}^{2}\right)$ & $2.13(0.60)$ & $2.27(0.70$ & 0.052 \\
\hline Peak aortic velocity $(\mathrm{cm} / \mathrm{s})$ & $223(54.5)$ & $220(57.1)$ & 0.666 \\
\hline Peak aortic gradient $(\mathrm{mm} \mathrm{Hg})$ & $21.0(10.9)$ & $20.7(11.7)$ & 0.852 \\
\hline Mean aortic gradient $(\mathrm{mm} \mathrm{Hg})$ & $12.5(6.7)$ & $12.1(7.2)$ & 0.677 \\
\hline Ejection fraction (\%) & $62.7(11.6)$ & $59.4(13.8)$ & 0.017 \\
\hline Stroke volume $(\mathrm{mL})$ & $92.3(25.9)$ & $97.2(29.9)$ & 0.133 \\
\hline Moderate aortic regurgitation (\%) & 5 & 5 & 1.000 \\
\hline Moderate mitral regurgitation (\%) & 12 & 7 & 0.102 \\
\hline Severe mitral regurgitation (\%) & 3 & 1 & 0.145 \\
\hline \multicolumn{4}{|l|}{1 year post-TAVR } \\
\hline Aortic valve area (VTI) $\left(\mathrm{cm}^{2}\right)$ & $1.92(0.59)$ & $2.13(0.60)$ & 0.045 \\
\hline Peak aortic velocity $(\mathrm{cm} / \mathrm{s})$ & $224(53.0)$ & $215(43.1)$ & 0.363 \\
\hline Peak aortic gradient $(\mathrm{mm} \mathrm{Hg})$ & $21.5(11.0)$ & $19.2(8.2)$ & 0.192 \\
\hline Mean aortic gradient $(\mathrm{mm} \mathrm{Hg})$ & $12.4(6.5)$ & $10.6(4.4)$ & 0.0696 \\
\hline Ejection fraction (\%) & $57.6(13.9)$ & $59.0(11.2)$ & 0.509 \\
\hline Stroke volume $(\mathrm{mL})$ & $93.8(28.8)$ & $92.9(27.9)$ & 0.862 \\
\hline Moderate aortic regurgitation (\%) & 12 & 15 & 0.612 \\
\hline Moderate mitral regurgitation (\%) & 14 & 9 & 0.602 \\
\hline Severe mitral regurgitation $(\%)$ & 9 & 0 & 0.051 \\
\hline
\end{tabular}

Values are mean (SD) or \%.

BP, blood pressure; TAVR, transcatheter aortic valve replacement; VTI, velocity time integral.

with new onset or worsening HTN had significantly better survival than non-hypertensive patients up to 2 years after TAVR (table 4). Baseline, immediate postprocedural, and 1-year HTN and antihypertensive medication data are given in table 5. Overall antihypertensive medications, particularly beta-adrenergic blockers, were utilised at a high rate in both study groups, with a statistically significant difference in the mean number of antihypertensive medication at 1 year.

\section{DISCUSSION}

This study suggests an association between the development or exacerbation of HTN and improved survival up to 2 years after TAVR. The improvement in survival may reflect the presence of myocardial contractile reserve, improvement in ventricular compliance and diastolic function in the setting of decreased arterial compliance associated with advanced age and longstanding HTN. A lack of a hypertensive response to TAVR may correlate with poor myocardial contractile reserve, myocardial fibrosis and fixed restrictive diastolic dysfunction. These hypotheses warrant further study since the presence or absence of these conditions may assist in determining those who may benefit most from TAVR.

The data from this study also demonstrate an observed mortality benefit in patients with new or exacerbated HTN in regard to inhospital mortality. The causative factors for this are uncertain, that is there were no differences in hospital HF exacerbations, pacemaker dependence or arrhythmias between the two groups. Furthermore, there were no significant differences in echocardiographic parameters directly after the procedure which would explain this difference. We believe that this is evidence of dormant myocardial reserve and suggests that there may be a 'window period' in which TAVR is most protective of the cardiac myocardium. This demonstrated benefit may indicate that the lack of an increase in afterload after TAVR suggest that there exists little preservable myocardium in some patients after TAVR. It may also suggest that the patients with increased BP differ only from the control group in this study in that the increased BP cohort was intervened on before myocardial remodelling became significant. We speculate that further improvements in TAVR outcomes could be made through earlier intervention in more closely monitored patients before TAVR and may indicate a role for cardiac MRI (cMRI) in pre-TAVR patient selection. The superior spatial resolution of cMRI over other imaging modalities may help identify patients with little subendocardial fibrosis, who potentially would have the best response to TAVR. ${ }^{20}$

Correspondingly, patients who lack a hypertensive response may benefit from a more intensive cardiac rehabilitation 


\begin{tabular}{|c|c|c|c|}
\hline & Increased BP & Stable BP & $P$ value \\
\hline$\%$ Survival $>1$ month & $99(192 / 193)$ & $88(132 / 150)$ & $<0.001$ \\
\hline$\%$ Survival $>6$ month & $96(152 / 159)$ & $79(92 / 116)$ & $<0.001$ \\
\hline$\%$ Survival $>1$ year & $89(116 / 130)$ & $67(65 / 97)$ & $<0.001$ \\
\hline$\%$ Survival $>2$ year & $72(59 / 82)$ & $46(30 / 65)$ & 0.002 \\
\hline Periprocedural major vascular & $14(7)$ & $15(10)$ & 0.435 \\
\hline Periprocedural minor vascular & $15(8)$ & $15(10)$ & 0.564 \\
\hline Post-TAVR PPM implantation & $18(9)$ & $10(7)$ & 0.430 \\
\hline \multicolumn{4}{|l|}{ Inhospital } \\
\hline CV mortality & $1(1)$ & $18(12)$ & $<0.001$ \\
\hline MI & $1(1)$ & $1(1)$ & 1.000 \\
\hline Stroke/TIA & $3(2)$ & $6(4)$ & 0.187 \\
\hline HF exacerbation & $36(19)$ & $37(25)$ & 0.186 \\
\hline \multicolumn{4}{|l|}{ Discharge to 30 days } \\
\hline MACCE & $36(19)$ & $21(16)$ & 0.554 \\
\hline CV mortality & $0(0)$ & $1(1)$ & 0.410 \\
\hline Myocardial Infraction & $2(1)$ & $2(2)$ & 1.000 \\
\hline Stroke/TIA & $2(1)$ & $1(1)$ & 1.000 \\
\hline HF exacerbation & $32(17)$ & $19(14)$ & 0.642 \\
\hline Rehospitalisation For any reason & $35(18)$ & $21(16)$ & 0.654 \\
\hline \multicolumn{4}{|l|}{30 days -6 months } \\
\hline MACCE & $45(29)$ & $24(25)$ & 0.472 \\
\hline CV mortality & $4(3)$ & $1(1)$ & 0.652 \\
\hline MI & $1(1)$ & $3(3)$ & 0.160 \\
\hline Stroke/TIA & $4(3)$ & $2(2)$ & 1.000 \\
\hline HF exacerbation & $30(19)$ & $8(8)$ & 0.018 \\
\hline Rehospitalisation for any reason & $44(28)$ & $19(20)$ & 0.137 \\
\hline \multicolumn{4}{|l|}{6 months -1 year } \\
\hline MACCE & $39(34)$ & $17(25)$ & 0.189 \\
\hline CV mortality & $3(3)$ & $1(1)$ & 1.000 \\
\hline MI & $3(3)$ & $1(1)$ & 1.000 \\
\hline Stroke/TIA & $2(2)$ & $1(1)$ & 1.000 \\
\hline HF exacerbation & $33(29)$ & $2(3)$ & $<0.001$ \\
\hline Rehospitalisation for any reason & $37(32)$ & $14(20)$ & 0.090 \\
\hline
\end{tabular}

Values are $\%$ (n).

$\mathrm{BP}$, blood pressure; CV, cardiovascular; HF, heart failure; MACCE, major adverse cardiovascular and cerebrovascular events, defined as death from any cause, MI, rehospitalisation and stroke; MI, myocardial infarction; PPM, permanent pacemaker; TAVR, transcatheter aortic valve replacement; TIA, transient ischaemic attack.

programme following TAVR. Regular BP monitoring may be appropriate to risk stratify patients post-TAVR, identifying those at risk for adverse outcomes. Intensification of HF treatment and cardiac rehabilitation in higher risk patients may lead to an improvement in functional status, quality of life, reduce hospitalisation and mortality after TAVR. ${ }^{21}$

Optimal management of HTN after TAVR is unclear. Aggressive BP management has the potential to increase adverse events in this high-risk population. Adding to this complexity, the recent SPRINT trial and JNC8 guidelines provide conflicting evidence on the benefits and risks of intensive BP lowering in elderly and frail patients. ${ }^{22} 23$ Based on our study, we believe that allowing for moderately increased BP targets may be the most prudent approach after TAVR to avoid the risks of hypotension.

This study also uncovered an association between post-TAVR HTN and HF exacerbations which is the first documented postprocedural risk factor for $\mathrm{HF}$ exacerbation in this patient population. The specific aetiological factors for this increase is unknown, but is likely secondary to uncontrolled HTN and challenges in medication titration in the post-TAVR time period. Previous studies have indicated that uncontrolled HTN may be the causative factor in up to $13 \%$ of HF exacerbations in patients with reduced ejection fraction and $16 \%$ in cases of preserved ejections factors. ${ }^{24}$ This uncontrolled HTN may also lead to subclinical myocardial ischaemia and an increased propensity for atrial fibrillation, which may further predispose patients who underwent TAVR to HF exacerbations. ${ }^{25}$

Still, the relationship between TAVR, cardiac haemodynamics and heart exacerbations remains uncertain. In a study by Chrissoheris et al it was demonstrated that TAVR is associated with improvements in stroke volume, cardiac output and central venous pressure in the first 24 hours after TAVR. ${ }^{26}$ Our study did not show these same significant improvement which draws into question the sustainability the acute changes in cardiac haemodynamic noted in this study. It would appear that controlling BP and blunting the acute and long-term changes in cardiac haemodynamics may be critical in reducing heart exacerbations and hospitalisations.

This study is limited by its retrospective design, single-centre experience and inequalities in the length of post-TAVR follow-up. The potential for confounding factors which were not identified and addressed in the study's baseline patient characteristics does exist. Patients in both groups were well matched overall, except that the increased BP group had statistically more female patients 
Table 5 HTN and antihypertensive data

\begin{tabular}{|c|c|c|c|}
\hline & Increased BP & Stable BP & $P$ value \\
\hline Mean preprocedural systolic BP (mm Hg) & $125.1(17.8)$ & $120.2(16.1)$ & 0.180 \\
\hline Mean preprocedural diastolic BP (mm Hg) & $67.0(10.2)$ & $67.0(10.3)$ & 0.910 \\
\hline Mean preprocedural pulse pressure $(\mathrm{mm} \mathrm{Hg})$ & $58.2(16.1)$ & $53.9(13.3)$ & 0.006 \\
\hline Mean \# of preprocedural BP meds & $2.23(1.08)$ & $2.41(1.17)$ & 0.150 \\
\hline ACE inhibitor (\%) & 31 & 35 & 0.415 \\
\hline Angiotensin II receptor blocker (\%) & 18 & 18 & 1.000 \\
\hline Beta blocker (\%) & 70 & 79 & 0.083 \\
\hline Calcium channel blocker (\%) & 27 & 31 & 0.470 \\
\hline Thiazide diuretic (\%) & 21 & 21 & 1.000 \\
\hline Loop diuretic (\%) & 48 & 50 & 0.827 \\
\hline Spironolactone (\%) & 4 & 3 & 1.000 \\
\hline Mean post-TAVR systolic BP $(\mathrm{mm} \mathrm{Hg})$ & $125.2(18.7)$ & $123.2(20.1)$ & 0.350 \\
\hline Mean post-TAVR diastolic BP (mm Hg) & $59.6(15.0)$ & $59.0(14.3)$ & 0.713 \\
\hline Mean post-TAVR pulse pressure $(\mathrm{mm} \mathrm{Hg})$ & $65.6(18.2)$ & $64.2(18.3)$ & 0.500 \\
\hline Mean \# of BP meds at 1 year post-TAVR & $2.77(0.92)$ & $2.19(1.06)$ & $<.001$ \\
\hline ACE inhibitor (\%) & 39 & 27 & 0.176 \\
\hline Angiotensin II receptor blocker (\%) & 22 & 20 & 1.000 \\
\hline Beta blocker (\%) & 88 & 81 & 0.250 \\
\hline Calcium channel blocker (\%) & 24 & 20 & 0.702 \\
\hline Thiazide diuretic (\%) & 20 & 17 & 0.687 \\
\hline Loop diuretic (\%) & 65 & 47 & 0.034 \\
\hline Spironolactone (\%) & 11 & 2 & 0.036 \\
\hline
\end{tabular}

Values are mean (SD) or \%.

BP, blood pressure; HTN, hypertension; TAVR, transcatheter aortic valve replacement.

and a greater utilisation of the first-generation Edwards Sapien valve. There is conflicting evidence on the impact of female sex on the outcomes of TAVR, and this could have influenced the findings of our study. 27

\section{Key messages}

\section{What is already known about this subject?}

- Previous studies have found that a transcatheter aortic valve replacement (TAVR) is associated with an increase in blood pressure (BP) in about half of patients after the procedure. This has been associated with a paradoxical improvement in long and intermediate outcomes including overall survival and procedural outcomes.

What does this study add?

- This study is important because it adds to what is previously known about the relationship between BP and TAVR and extends out the period of monitoring beyond 1 year postTAVR.

- This study also provides important data on the potential longterm impact of TAVR on echocardiographic parameters.

- Furthermore, this study is the first to associated increased BP after TAVR and an increased risk in heart failure (HF) exacerbations in the first year following TAVR.

How might this impact on clinical practice?

- This study helps to establish a pragmatic way of risk stratifying patients for outpatient follow-up after TAVR.

- It is also the first study to associated hypertension (HTN) after TAVR and an increase risk in HF exacerbations, which could also serve as a guide for outpatient monitoring and pharmacological management of post-TAVR HTN. These findings could be an important first step in redesigning outpatient follow-up for patients after TAVR.

\section{CONCLUSION}

In this study, an association between the development or worsening of HTN after TAVR and improved clinical outcomes, most notably increased overall survival, was found. Additionally, the development or worsening of HTN after TAVR was also associated with a statistically significant increase in postprocedural HF exacerbations. The association between BP and post-TAVR mortality could lead to further study of methods to risk stratify patients who underwent TAVR in the preoperative and postoperative period.

Acknowledgements The investigators would like to thank Abe Sahmoun, PhD, for his help with the statistical analysis for this study and Ronda Bolgrean, RN, for her help with data acquisition.

Contributors BJK was involved and primarily responsibly for all phases of this research and manuscript. CMD and TAH were involved in data interpretation and critical revision of manuscript.

Funding The authors have not declared a specific grant for this research from any funding agency in the public, commercial or not-for-profit sectors.

Competing interests TAH is on the Speaker's Bureau on Astra Zeneca.

Patient consent Not required.

Ethics approval Univeristy of North Dakota IRB and Sanford Health IRB.

Provenance and peer review Not commissioned; externally peer reviewed.

(C) Article author(s) (or their employer(s) unless otherwise stated in the text of the article) 2018. All rights reserved. No commercial use is permitted unless otherwise expressly granted.

\section{REFERENCES}

1 Makkar RR, Fontana GP, Jilaihawi H, et al. Transcatheter aortic-valve replacement for inoperable severe aortic stenosis. N Engl J Med 2012;366:1696-704.

2 Arnold SV, Reynolds MR, Wang K, et al. Health status after transcatheter or surgical aortic valve replacement in patients with severe aortic stenosis at increased surgical risk: results from the corevalve US pivotal trial. JACC Cardiovasc Interv 2015:8:1207-17.

3 Smith CR, Leon MB, Mack MJ, et al. Transcatheter versus surgical aortic-valve replacement in high-risk patients. N Eng/ J Med 2011;364:2187-98. 
4 Arnold SV, Reynolds MR, Lei Y, et al. Predictors of poor outcomes after transcatheter aortic valve replacement: results from the PARTNER (Placement of Aortic Transcatheter Valve) trial. Circulation 2014;129:2682-90.

5 Konigstein M, Ben-Assa E, Abramowitz Y, et al. Usefulness of updated valve academic research consortium-2 criteria for acute kidney injury following transcatheter aortic valve implantation. Am J Cardiol 2013;112:1807-11.

6 Kodali SK, Williams MR, Smith CR, et al. Two-year outcomes after transcatheter or surgical aortic-valve replacement. N Engl J Med 2012;366:1686-95.

7 Anjan VY, Herrmann HC, Pibarot P, et al. Evaluation of flow after transcatheter aortic valve replacement in patients with low-flow aortic stenosis: a secondary analysis of the PARTNER randomized clinical trial. JAMA Cardiol 2016;1:584-92.

8 Généreux P, Cohen DJ, Mack M, et al. Incidence, predictors, and prognostic impact of late bleeding complications after transcatheter aortic valve replacement. J Am Coll Cardiol 2014;64:2605-15.

9 Ussia GP, Barbanti M, Petronio AS, et al. Transcatheter aortic valve implantation: 3-year outcomes of self-expanding CoreValve prosthesis. Eur Heart J 2012;33:969-76.

10 Paradis JM, Maniar HS, Lasala JM, et al. Clinical and functional outcomes associated with myocardial injury after transfemoral and transapical transcatheter aortic valve replacement: a subanalysis from the PARTNER Trial (Placement of Aortic Transcatheter Valves). JACC Cardiovasc Interv 2015;8:1468-79.

11 Zahn R, Gerckens U, Linke A, et al. Predictors of one-year mortality after transcatheter aortic valve implantation for severe symptomatic aortic stenosis. Am J Cardiol 2013;112:272-9.

12 Tamburino C, Capodanno D, Ramondo A, et al. Incidence and predictors of early and late mortality after transcatheter aortic valve implantation in 663 patients with severe aortic stenosis. Circulation 2011;123:299-308.

13 Miller DC, Blackstone EH, Mack MJ, et al. Transcatheter (TAVR) versus surgical (AVR) aortic valve replacement: occurrence, hazard, risk factors, and consequences of neurologic events in the PARTNER trial. J Thorac Cardiovasc Surg 2012;143:832-43.

14 Sinning JM, Scheer AC, Adenauer V, et al. Systemic inflammatory response syndrome predicts increased mortality in patients after transcatheter aortic valve implantation. Eur Heart J 2012;33:1459-68.
15 Maan A, Heist EK, Passeri J, et al. Impact of atrial fibrillation on outcomes in patients who underwent transcatheter aortic valve replacement. Am J Cardiol 2015;115:220-6.

16 Nazif TM, Dizon JM, Hahn RT, et al. Predictors and clinical outcomes of permanent pacemaker implantation after transcatheter aortic valve replacement. JACC Cardiovasc Interv 2015;8:60-9.

17 Perlman GY, Loncar S, Pollak A, et al. Post-procedural hypertension following transcatheter aortic valve implantation: incidence and clinical significance. JACC Cardiovasc Interv 2013;6:472-8.

18 Lindman BR, Otto CM, Douglas PS, et al. Blood pressure and arterial load after transcatheter aortic valve replacement for aortic stenosis. Circ Cardiovasc Imaging 2017;10:e006308.

19 Kappetein AP, Head SJ, Généreux P, et al. Updated standardized endpoint definitions for transcatheter aortic valve implantation: the Valve Academic Research Consortium-2 consensus document. J Thorac Cardiovasc Surg 2013;145:6-23.

20 Partington SL, Kwong RY, Dorbala S. Multimodality imaging in the assessment of myocardial viability. Heart Fail Rev 2011;16:381-95.

21 Zanettini R, Gatto G, Mori I, et al. Cardiac rehabilitation and mid-term follow-up after transcatheter aortic valve implantation. J Geriatr Cardiol 2014;11:279-85.

22 Williamson JD, Supiano MA, Applegate WB, et al. Intensive vs standard blood pressure control and cardiovascular disease outcomes in adults aged $\geq 75$ years: a randomized clinical trial. JAMA 2016;315:2673-82.

23 James PA, Oparil S, Carter BL, et al. 2014 evidence-based guideline for the management of high blood pressure in adults: report from the panel members appointed to the Eighth Joint National Committee (JNC 8). JAMA 2014;311:507-20.

24 Kapoor JR, Kapoor R, Ju C, et al. Precipitating clinical factors, heart failure characterization, and outcomes in patients hospitalized with heart failure with reduced, borderline, and preserved ejection fraction. JACC Heart Fail 2016;4:464-72.

25 Mitchell GF, Vasan RS, Keyes MJ, et al. Pulse pressure and risk of new-onset atrial fibrillation. JAMA 2007;297:709-15.

26 Chrissoheris M, Ziakas A, Chalapas A, et al. Acute invasive hemodynamic effects of transcatheter aortic valve replacement. J Heart Valve Dis 2016;25:162-72.

27 Chandrasekhar J, Dangas G, Yu J, et al. Sex-based differences in outcomes with transcatheter aortic valve therapy: TVT registry from 2011 to 2014. J Am Coll Cardiol 2016;68:2733-44. 